\title{
TECHNOLOGIES BASED ON NEW SURFACTANTS FOR WASTEWATER TREATMENT
}

\author{
Ciprian Chelaru 1 , Marius Simion², Dana Varasteanu ${ }^{3}$, Irina Chican ${ }^{3}$
}

\author{
${ }^{1}$ INCDTP- Division ICPI, 93, Ion Minulescu, 031215, sector 3, Bucharest, \\ Romania \\ 2 The National Research-Development Institute for Industrial Ecology-ECOIND, \\ 71-73 Drumul Podu Dambovitei str., district 6, Bucharest, Romania \\ 3 The National Institute for Research \& Development in Chemistry and \\ Petrochemistry -ICECHIM, 202 Spl. Independentei str., Bucharest, Romania
}

\section{Abstract}

There is a trend to select, characterize and use new types of surfactants for phase separation processes and developing membranary technologies (e.g. emulsion and liquid membranes) with high yield in removal of: organic substances, metals, dyes, etc, from wastewater.

New surfactants are improved continuously, as required because of the dynamics of user technologies and of the indispensability of environmental protection through selection of raw materials without adversely affecting the environment.

The paper provides new technological solutions for separation of pollutants from wastewater using gemini and bolaamfifile surfactants, with creation self assembling structures. These new self assembling nanostructures obtained are used in the technology of separation emulsion membranes. Novel technologies based on surfactants are effective, fast, have a convenient price, not require complex utilities, ecologic because reduced the negative environmental impact.

Due to specific properties such as micellization, solubility, solubilization, surface activity, surfactants intervene in the membrane production process, in the pre-treatment and their characterization respectively but also in the membrane separation processes (filtration, micellar ultrafiltration) of organic compounds (dyes, intermediaries, phenols, proteins) and metal ions.

Keywords: New self assembling nanostructures, surfactants, emulsion and liquid membranes, pollutants

\section{Introduction}

Surfactants are used both globally and in the country, in practically all branches of economic activity: petroleum extraction and processing (oil demulsifiers, fluids for secondary and tertiary recovery of petroleum), extractive industry (ore flotation), nuclear energetics, construction, metal processing (emulsifiable oils, corrosion inhibitors, lubrication and cooling liquids, nondestructive defectoscopy, washing, degreasing, metal coating agents), plastics, agriculture (emulsifiable concentrates, wettable grains, emulsions, encapsulated products) and medicines. 
The functional properties that surfactants manifest are: wetting, moistening, emulsifying, demulsifying, solubilization, lubrication, anticorrosive protection. Surfactants can participate in a large number of separation processes bringing their contribution in different ways: in membrane processes, sedimentation processes, centrifugation, electrophoresis, magnetic separation, flotation, chromatography, phase separation, extraction.

Surfactants have a multiple role:

- conditioning active substances specific to each application field, through the solubilization and emulsifying effect, leading to the occurrence of stable disperse systems (colloidal);

- conferring specific properties: wetting, hydrophilization, hydrophobization to: solution-surfactant interface, solid surfaces, plastics, rubber, inorganic and organic grains and powders, favors processes of transport through emulsion membranes;

- achieving synergistic effects mixed with other active ingredients such as lubrication, anticorrosive protection, sustainability of liquid film.

A special type of surfactants is the class of bolaamphiphiles (bolaform amphiphiles, bolaphiles, bolas, etc.). They consist of a hydrophobic spacer in the central part of the molecule (which may contain one, two or several alkyl, steroid or porphyrin chains, etc.) and two identical or different hydrophilic groups at the ends of the hydrophobic spacer. Bolaamphiphiles have the ability to self-organize at water/air or water/solvent interfaces. In solution they can selfassemble in structures such as vesicles, fibers, ribbons, tubules, planar monolayers and multilayers, etc. depending on various factors [1-8].

Compared with classic tensides, the introduction of a second hydrophilic group to the bolaamphiphiles increases their water solubility and the critical micellar concentration, while decreases the aggregation number.

The bolaforms can find many applications. They might be used as template in the synthesis of nanomaterials, in sensors manufacturing, developing nanotubes with applications in electronics, fabrication of core-shell structures for drug delivery in medicine, or in catalysis applications.

A special class of surfactants nanoarchitectures is represented by multisheets nanostructures organized as flower-like assemblies. Such nanoarchitectures were reported, for example, in the polymerization process of aniline where nanosheets stack layer-by-layer onto multisheets architectures like flower petals. These hierarchical architectures have been obtained using an amphiphilic amino acid, valine, as dopant in aniline polymerization or other amino acids such as threonine, proline and arginine. Their properties made them appropriate for preparation of superhydrophobic surfaces.

Also, like bolaforms, in solution are self-assembly an giant gemini surfactant consisting of two hydrophilic carboxylic acid-functionalized polyhedral oligomeric silsesquioxane (APOSS) heads and two hydrophobic polystyrene (PS) tails covalently linked via a rigid spacer ( $p$-phenylene or biphenylene) (PS(APOSS) $2-P S$ ), [9]. Their self-assembly in solution revealed a morphological transition from vesicles to wormlike cylinders and further to spheres as the degree of ionization of the carboxylic acid groups on POSS heads increases. It was found that the PS tails are generally less stretched in the micellar cores of 
these giant gemini surfactant than those of the corresponding single-tailed (APOSS-PS) giant surfactant. It was further observed that the PS tail conformations in the micelles were also affected by the length of the rigid spacers where the one with longer spacer exhibits even more stretched PS tail conformation. Both findings could be explained by the topological constraint imposed by the short rigid spacer in PS-(APOSS) 2 -PS gemini surfactant. This constraint effectively increases the local charge density and leads to an anisotropic head shape that requires a proper re-distribution of the APOSS heads on the micellar surface to minimize the total electrostatic repulsive free energy. The study expands the scope of giant molecular shape amphiphiles and has general implications in the basic physical-chemical principles underlying their solution self-assembly behaviors as nanostructures.

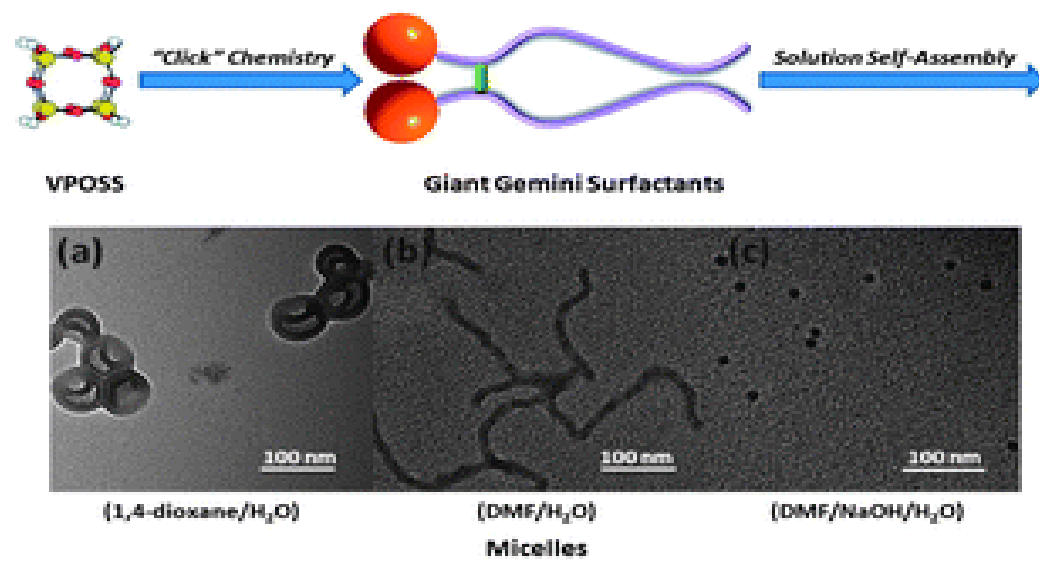

Fig.1. Illustration of self-assembly in nanostructures of an giant gemini surfactant, consisting of two hydrophilic carboxylic acid-functionalized polyhedral oligomeric silsesquioxane (APOSS) heads and two hydrophobic polystyrene (PS) tails covalently linked via a rigid spacer ( $p$-phenylene or biphenylene) (PS-(APOSS) $\left.{ }_{2}-\mathrm{PS}\right),[9]$

In this paper new self assembling nanostructures (flower-like association) were developed using bolaamfifile surfactants in different systems and is the possibility to used in separation processes or membranary technologies (e.g. emulsion and liquid membranes) for removal of: organic substances, metals, dyes, etc, from wastewater. The morphology of flower-like association complexes were noticed in the systems with dodecandioyl-diglycine bolaamphiphile/ polymer/solvent.

This new surfactants (gemini and bola) self assembling nanostructures we used in he technology of separation emulsion membranes.

\section{Materials and methods}

The experimental techniques used in the study consist in ultraviolet, infrared spectral methods, electronic scanning microscopy, chromatography, dynamic light scattering:

- Scanning electron microscopy was carried out with an equipment type "SEM QUANTA 200" from FEI company, with EDAX; 
- We used an UV-VIS spectrophotometer JASCO, model 500, with quartz cuvettes of $1 \mathrm{~cm}^{3}$ and layer thickness in the range $200 \mathrm{~nm}-800 \mathrm{~nm}$;

- Dynamic light scattering using equipment zetasizer-nano type "Malvern", with measuring range between $0.3 \mathrm{~nm}-10.0$ microns and zeta potential determination with an accuracy of $+/-2 \%$;

- High performance liquid chromatography was performed with an equipment Jasco Lcenet II, with autosampler, wavelength in the range: 200-700 nm.

\section{Results and Discussion}

The first step in the investigation was to determine the critical concentrations of the bola surfactant, dodecandioyl-diglycine (Bola):

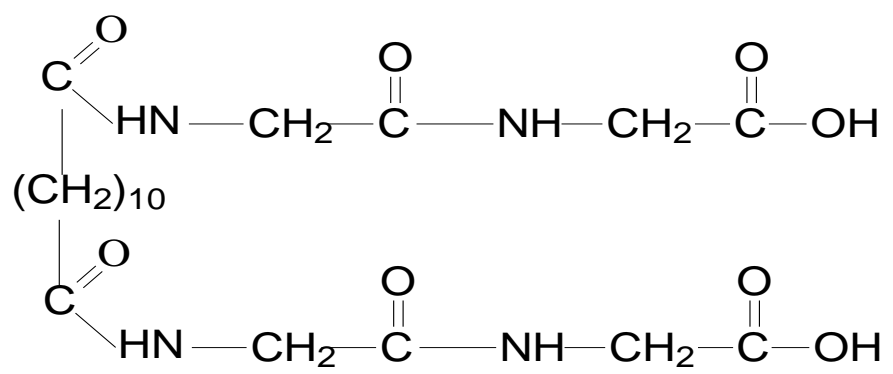

and gemini (Gemini) with $\mathrm{n}=2$ :

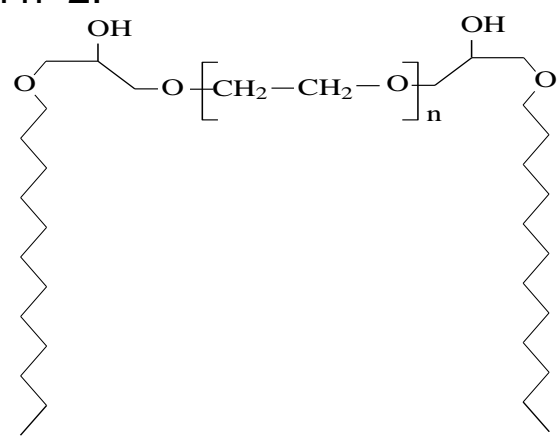

where self-assembly forms appear.

UV-VIS spectroscopic method was used for setting off the critical concentrations of gemini and bola surfactants. If the surfactant shows UV-VIS absorption, the molar absorptivity suddenly changes at critical concentration which explains modification of the slope of the absorbance vs concentration plot.

However, no CMC could be found for bolaform in water using the above mentioned UV-VIS method although self-assembling structures were revealed by SEM. The association forms for these types of bolaform are vesicles not micelles like for classic tensides.

It was used these gemini and bola surfactants and their nanostructures: vesicles, micelles or flower-like in membranes technologies.

Membrane processes have known a spectacular development starting from the early $70^{\text {s }}$, being used at industry level in fields such as: wastewater treatment, medical technology, chemical industry. Rapid and diverse evolution 
of these technologies was made possible by perfecting experimental techniques for preparation and characterization of membranes as well as by the numerous advantages it offers:

- lower energy consumption of the separation process;

- the possibility of continuous operation;

- simple operating conditions;

- possibilities of automatization;

- possibility of coupling membrane processes with classic separation procedures;

- flexibility in their potential applications.

The technology of separation emulsion membranes is relatively recent and has drawn the attention of specialists, due to the advantages it offers compared to solid membranes or liquid extraction.

Achieving separation using emulsion membranes involves a process resulting from the following stages: preparation of water/oil type emulsion, emulsion contact, for a limited time, with the source phase, namely the formation of double water/oil/water emulsion with the receiving phase; emulsion separation and destruction, with the recovery of film former organic solvent, as well as of the aqueous solution containing transported permeation.

Choosing the receiving aqueous phase and organic solvent is determined by the properties of chemical substance to be separated, and the nature and concentration of the emulsifying agent, the receiving aqueous phase/organic phase ratio and mixing shall be determined on the basis of technical and economic considerations, which make the process accessible at a desired or required scale.

Multiple emulsions are complex systems in which the droplets of the dispersed phase contain a continuous phase of other dispersed droplets.

The main types of multi-emulsions are water-in-oil-in-water $(\mathrm{W} / \mathrm{O} / \mathrm{W})$ and the oil-in-water-in-oil (W/O/W).

In most cases, multiple emulsions were prepared using a two-step emulsification process, fig.2.

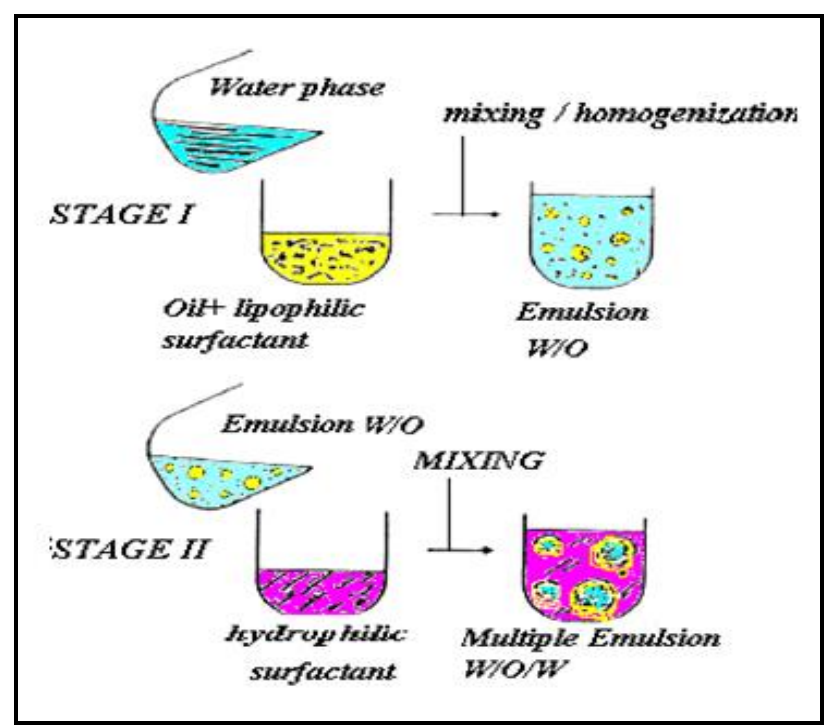

Fig.2. Schematic illustration of the two-step emulsification process 
Two types of emulsifiers were used: gemini or bolaamfifile surfactants hydrophobic I (for the formation of the emulsion $\mathrm{W} / \mathrm{O}$ ) and the other hydrophilic II (for the emulsion $\mathrm{O} / \mathrm{W}$ ). Reverse micelle technology was used for obtaining nanostructures. The results and the: aspects, dimension, structure of nanostructures are shown in the fig.3, a-b.

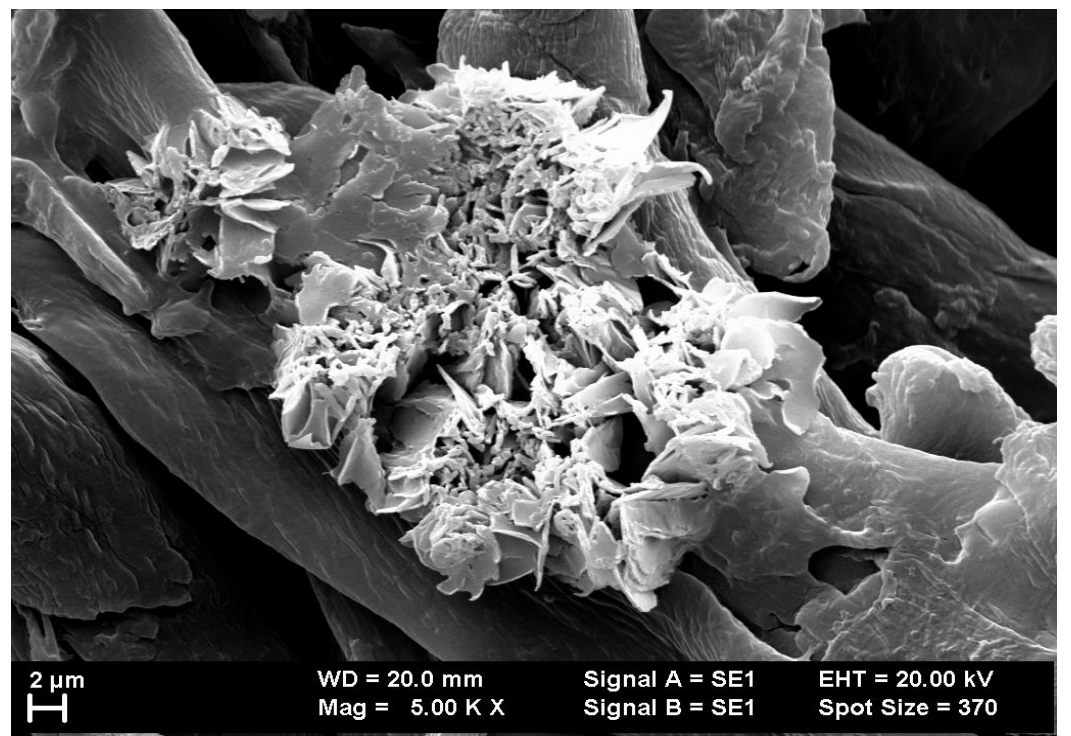

a)

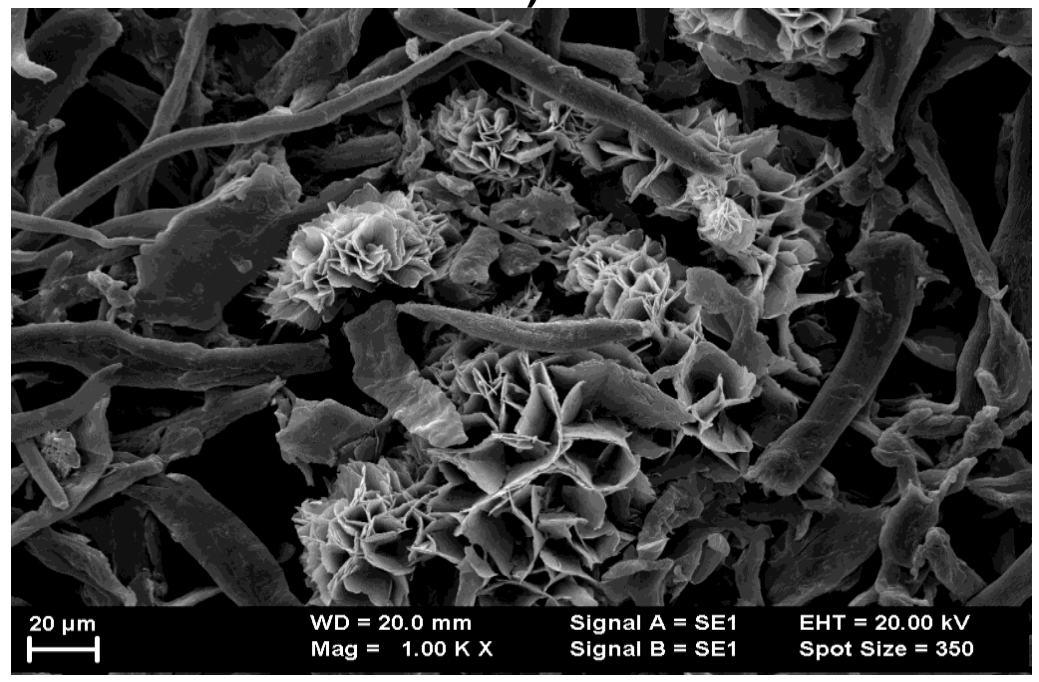

b)

Fig. 3. SEM micrographs for flower-like nanostructures in bola surfactant/polymer (cellulose) /solvent systems $(a, b)$

The nanosheet microstructures built as flower-like morphologies are the result of interaction of an polymer (cellulose) with glycine-based bolaamphiphile. Responsible for this multisheets morphology are the complex interactions which involve both hydrogen bonds and chemical reactions between bolaamphiphile surfactant and polymer microcrystals.

The nanostructures obtained, based from new surfactants (gemini and bolaform) are possible to used in membranary technologies for remove organic pollutants. 


\section{Conclusions}

The interaction of polymer with dodecandioyl-diglycine bolamphiphile results in a nano and microstructured flower-like complex association and can used in removal of drugs or other organic substances with membranary technologies.

An application of these nanostructures with flower-like morphologies is retention of polar pollutants (e.g. some hormones, pesticides, etc.) very difficult to separate using membranary technologies.

Knowing and controlling the mechanism of pollutants immobilization in new self assembling nanostructures of surfactants and used them in membranary technologies is a protection solution for environment.

\section{References}

[1] Nagarajan R. Polymer-Surfactant Interactions. In: "New Horizons: Detergents for the New Millennium Conference Invited Papers", American Oil Chemists Society and Consumer Specialty Products Association Ed.: Fort Myers: Florida; 2001.

[2] Fuhrhop JH, Wang T. Bolaamphiphiles. Chemical Reviews 2004; 104: 2901 38.

[3] Meister A, Blume A. Self-assembly of bipolar amphiphiles. Current Opinion in Colloid \& Interface Science 2007;12:138-47.

[4] Infante MR, Pérez L, Pinazo A, Clapés P, Morán MC, Angelet M, García MT, Vinardell MP. Amino acid-based surfactants. C. R. Chimie 2004; 7:583-92.

[5] Matsui H, Gologan B. Crystalline Glycylglycine Bolaamphiphile Tubules and Their pH-Sensitive Structural Transformation. The Journal of physical chemistry B 2000; 104(15): 3383-6.

[6] Jinyoung K, Sang YL. pH-sensitive transformation of the peptidic bolaamphiphile self-assembly: Exploitation for the $\mathrm{pH}$-triggered chemical reaction. Colloids and Surfaces B, Biointerfaces 2014; 115:406-11.

[7] Nurxat N, Hanying B, Kai S. Bolaamphiphilic molecules: Assembly and applications. Progress in Polymer Science 2013; 38: 302-43.

[8] Kogiso M, Ohnishi S, Yase K, Masuda M, Shimizu T. Dicarboxylic Oligopeptide Bolaamphiphiles: Proton-Triggered Self-Assembly of Microtubes with Loose Solid Surfaces. Langmuir 1998; 14: 4978-4986.

[9] Zhao Wang, Yiwen Li, Xue-Hui Dong, Xinfei Yu, Kai Guo, Hao Su, Kan Yue, Chys Wesdemiotis, Stephen Z. D. Cheng, Wen-Bin Zhang. Giant gemini surfactants based on polystyrene-hydrophilic polyhedral oligomeric silsesquioxane shape amphiphiles: sequential "click" chemistry and solution self-assembly. Chem. Sci. 2013; 4: 1345-1352; DOI: 10.1039/C3SC22297G 